\title{
LIX. On the theory of the transmission of power by dynamo-electrical machines
}

\section{Prof. R. Clausius}

To cite this article: Prof. R. Clausius (1884) LIX. On the theory of the transmission of power by dynamo-electrical machines, Philosophical Magazine Series 5, 17:109, 518-530, DOI: 10.1080/14786448408627552

To link to this article: http://dx.doi.org/10.1080/14786448408627552

曲 Published online: 29 Apr 2009.

Submit your article to this journal $[\pi$

Џll Article views: 4

Q View related articles $₫$ 
Thus if $p_{0}^{\prime}$ denote prob. that it happens in none, $p_{1}^{\prime}$ in one, $p^{\prime}{ }_{e}$ in $e$ times,

$$
\begin{gathered}
p_{0}^{\prime}: p_{1}^{\prime}: p_{2}^{\prime}: \ldots p_{e}^{\prime} \ldots p_{r}^{\prime} \\
=1: r: \frac{r \cdot(r-1)}{\mid 2}: \ldots \ldots \cdot \frac{r \cdot(r-1) \ldots(r-e+1)}{\mid e} \ldots: 1 .
\end{gathered}
$$

The ratio is that of the terms in the expansion of $(1+1)^{r}$.

Hence it is clear that, by the Theory of Probability, the occurrence of an event in the past tells us absolutely nothing as to its occurrence in the future. Also the larger the number of times we include in our expectation, the less should be our expectancy.

Our ignorance of the future is left therefore just what it was so far as any mere counting of cases in the past is concerned. The determination to be taught by facts, and facts only, to bring no preconceptions for the interpretation of these facts, cuts us off from all means of making advance to the new facts which are our quest. Presuppositions of knowledge, not of ignorance, are what we want. The latter is perfectly expressed in the statement that all constitutions of the Universe are equally possible. The principles of Inductive Science are the former. These assert that, so far from all constitutions of the Universe being equally possible, one only is-possible and also necessary. Hence our business in observing Nature is not the calculation of chances but the investigation of signs of order, the knowledge of which shall enable us to infer from them that one possible and necessary order of events.

LIX. On the Theory of the Transmission of Pover by Dynamo-electrical Machines. By Prof. R. Clausius*.

\$1. Equations which hold for individual Machines. TN a paper recently published $\dagger$ I deduced two equations 1 which serve to determine the work of the ponderomotive and of the electromotive force for a dynamo-electrical machine in operation, and which are as follows:-

$$
\begin{aligned}
& \mathrm{T}=-\left[\frac{1}{a+i}\left(p+\frac{q}{b+i}\right) v+\left(\frac{l}{a+i}\right)^{2}\left(\sigma+\frac{\lambda}{b+i}\right) v^{2}\right] i^{2}, \\
& \mathrm{E}=\left[\frac{1}{a+i}\left(p+\frac{q}{b+i}\right) v-\rho v-\left(\sigma+\frac{\lambda}{b+i}\right) v^{2}\right] i .
\end{aligned}
$$

In this $\mathrm{T}$ is the work of the ponderomotive force in unit of

* Translated from a separate impression, communicated by the Author, from the Amnalen der Physik und Chemie, Band xxi., 1884.

$\dagger$ Phil. Mag. anted, p. 46. 
time, and $\mathrm{E}$ the electromotive force; $i$ and $v$ represent the current-strength and the number of turns, while $a, b, p, q, l$, $\rho, \sigma$, and $\lambda$ are constants, of which those denoted by Greek letters, and especially the two last, are small as compared with the rest. If the two members in the first round bracket be reduced to a common denominator, and the sign $e$ be then introduced with the signification

$$
e=b+\frac{q}{p}, . . . . . . .
$$

the equations assume the following still simpler form :-

$$
\begin{aligned}
& \mathrm{T}=-\left[\frac{e+i}{(a+i)(b+i)} p v+\left(\frac{l}{a+i}\right)^{2}\left(\sigma+\frac{\lambda}{b+i}\right) v^{2}\right] i^{2}, . \\
& \mathrm{E}=\left[\frac{e+i}{(a+i)(b+i)} p v-\rho v-\left(\sigma+\frac{\lambda}{b+i}\right) v^{2}\right] i .
\end{aligned}
$$

These equations may be used in dealing with the transmission of force; that is, in treating the case in which two dynamo machines are in conducting communication, and one of them is driven by any mechanical motor, while the other is set in motion by the electrical current in the common conductor, and can thus do mechanical work. In order to make the application of the equations to this case as convenient as possible, it is advisable to introduce a little simplification in reference to the sign.

The second machine, which is set in motion by the current, rotates in the opposite direction to that which produces the current; and hence, if we wish to express this also in the equation, we must assign a negative value $v$ for the second machine. Further, the ponderomotive force is overcome in the motion of the first machine, while the second is set in motion by the ponderomotive force; hence if this also is to be expressed, we must represent the work $\mathrm{T}$ in the first machine by a negative magnitude, as has been done in the above equations; and in the second machine by a positive magnitude. Finally, the induced electromotive forces in the two machines are in opposite directions, and for a complete determination they also must be represented by positive and negative magnitudes.

But it would be inconvenient in practice to speak always of negative velocities of rotation in regard to one machine; and instead of this it is usual to express the velocities of rotation in both machines simply by the number of turns, and therefore by absolute magnitudes, and to regard it as a matter of course that the rotations are in opposite directions. It is 
also looked on as a matter of course that work is expended in one machine and is gained in the other, and that the electromotive forces induced in them are in opposite directions; and accordingly, in determining the values of the work and the electromotive forces, we are content with giving their absolute values. We shall follow this custom, and in accordance therewith shall represent by $v_{1}$ and $v_{2}$ the two numbers of turns without reference to the directions of rotations, by $T_{1}$ and $T_{2}$ the absolute. values of the work, and by $\mathrm{E}_{1}$ and $\mathrm{E}_{2}$ the absolute values of the electromotive forces.

The equations referring to the first machine are then as follows :-

$$
\begin{aligned}
& \mathrm{T}_{1}=\left[\frac{e+i}{(a+i)(b+i)} p v_{1}+\left(\frac{l}{a+i}\right)^{2}\left(\sigma+\frac{\lambda}{b+i}\right) v_{1}^{2}\right] i^{2}, . \\
& \mathrm{E}_{1}=\left[\frac{e+i}{(a+i)(b+i)} p v_{1}-\rho v_{1}-\left(\sigma+\frac{\lambda}{b+i}\right) v_{1}^{2}\right] i .
\end{aligned}
$$

In order to form the corresponding equations for the second machine, a remark must be made as to the sign of the members which have $v^{2}$ as factor, and of the member $\rho v$. Disregarding for the present the member $\rho v$, the following holds for the other members. If the signs of $\mathrm{T}$ and $\mathrm{E}$ are to be determined, then, in order to pass from the equations referring to the first machine to those which hold for the second machine, we must replace $v_{1}$ by $-v_{2}$. The members of the first order would thereby change their sign, while those of the second order would retain theirs unaltered. If, on the contrary, the absolute values only of $T$ and $E$ are to be determined, then in those cases in which the previous method would have given negative values of $T$ and $E$, we must change all signs on the right hand. The consequence of this would be that, in the equations which refer to the second machine, the members of the first order acquire the same sign as in the equations referring to the first machine, while those of the second degree acquire the opposite sign. As to the member $\rho v$, which we have hitherto disregarded, the electromotive force represented by the product $\rho v i$ acts always in that direction which is opposed to the direction of the current $i:$ hence in the first machine, in which the whole induced electromotive force has the direction of the current $i$, it must diminish its absolute value, which agrees with the minus sign prefixed to $\rho v$ (IV.); in the second machine, on the contrary, in which the whole electromotive force is opposed to the current $i$, the electromotive force represented by that product must increase the absolute value, and hence the member $\rho v$ must have the 
plus sign. Accordingly the equations assume the following form for the second machine:-

$$
\begin{aligned}
& \mathrm{T}_{2}=\left[\frac{e+i}{(a+i)(b+i)} p v_{2}-\left(\frac{l}{a+i}\right)^{2}\left(\sigma+\frac{\lambda}{b+i}\right) v_{2}^{2}\right] i^{2}, . \\
& \mathrm{E}_{2}=\left[\frac{e+i}{(a+i)(b+i)} p v_{2}+\rho v_{2}+\left(\sigma+\frac{\lambda}{b+i}\right) v_{2}^{2}\right] i .
\end{aligned}
$$

In forming these equations it is presupposed that the second machine is similar to the first. If the second machine is different from the first, the constants occurring in the equations, and which depend on the nature of the machine, would have different values from those which hold for the first machine; and this would have somehow to be indicated, for instance by prefixing indices to letters.

The four equations (III.), (IV.), (V.), (VI.) can now be used to make the calculations for the joint action of the two machines.

\section{§2. Deternination of the Current-strength.}

If the two machines are connected, as in the transmission of force, by conductors which contain no further electromotive force, the effective electromotive force in the whole circuit is represented by the difference $\mathrm{E}_{1}-\mathrm{E}_{2}$. Hence, if $\mathrm{R}$ is the resistance of the whole circuit, we may write

$$
\mathrm{R} i=\mathrm{E}_{1}-\mathrm{E}_{2} . \quad . \quad \cdot . \cdot .
$$

If we replace $E_{1}$ and $E_{2}$ in this, by their values from (IV.) and (VI.), we get

$$
\begin{aligned}
\mathrm{Ri} i=\left[\frac{e+i}{(a+i)(b+i)} p\left(v_{1}-v_{2}\right)-\rho\left(v_{1}+v_{2}\right)\right. \\
\left.-\left(\sigma+\frac{\lambda}{b+i}\right)\left(v_{1}^{2}+v_{2}^{2}\right)\right] i, .
\end{aligned}
$$

which equation is to be used for determining $i$.

As the first directly obvious solution we get

$$
i=0 \text {. . . . . . . . . }
$$

After this has been determined we may divide the equation by $i$, and after removing the members which have no denominator to the left hand, we get the following equation:-

$$
\begin{aligned}
\mathrm{R}+\rho & \left(v_{1}+v_{2}\right)+\sigma\left(v_{1}^{2}+v_{2}^{2}\right) \\
& =\frac{e+i}{(a+i)(b+i)} p\left(v_{1}-v_{2}\right)-\frac{\lambda}{b+i}\left(v_{1}^{2}+v_{2}^{2}\right) .
\end{aligned}
$$

This equation we imagine divided by the magnitude on 
the left hand, and introduce then the following abbreviated sigus :-

$$
\left.\begin{array}{l}
u=\frac{v_{1}-v_{2}}{\mathrm{R}+\rho\left(v_{1}+v_{2}\right)+\sigma\left(v_{1}^{2}+v_{2}^{2}\right)}, \\
\mathrm{U}=\frac{v_{1}^{2}+v_{2}^{2}}{\mathrm{R}+\rho\left(v_{1}+v_{2}\right)+\sigma\left(v_{1}^{2}+v_{2}^{2}\right)} \cdot
\end{array}\right\} .
$$

The equation then becomes.

$$
1=\frac{e+i}{(a+i)(b+i)} p u-\frac{\lambda}{b+i} \mathrm{U} . \text {. . . }
$$

If this equation be further multiplied by $(a+i)(b+i)$, and then the members arranged according to powers of $i$, we arrive at the quadratic equation

$$
i^{2}-(p u-\lambda U-a-b) i-e p u+\lambda a U+a b=0 ; .
$$

by solving which we obtain

$$
\begin{aligned}
i= & =\frac{1}{2}(p u-\lambda \mathrm{U}-a-b) \\
& \pm \frac{1}{2} \sqrt{(p u-\lambda \mathrm{U}-a-\bar{b})^{2}+4 e p u-4 \lambda a \mathrm{U}-4 a b .} .
\end{aligned}
$$

Of the two signs before the root we must choose the upper one, in accordance with my previous remark in reference to the corresponding equation; and by using this sign we must make the limitation that the expression only holds for such values of $u$ as are great enough to make it positive. For small values of $u$, below a certain limit, which make this expression negative, the value null for $i$, given in (4), must be taken to hold. If this is to be treated in all strictness we should have, in this interval, not the exact value null, but a very small value of $i$, depending on the remanent magnetism in the fixed electromagnet of the first machine. But as this interval only comprises those velocities of the frst machine which occur during the starting, and before the second machine is in motion, the very small values of $i$ within it are of small importance, and we need only direct our attention to the values which hold for greater velocities, and are defined by equation (9).

This equation may be put in a somewhat simpler form. If for the sake of shortness we put

$$
\begin{aligned}
& u^{\prime}=u-\frac{\lambda}{p^{\mathrm{q}}} \mathrm{U}=\frac{v_{1}-v_{2}-\frac{\lambda}{p}\left(v_{1}^{2}+v_{2}^{2}\right)}{\mathrm{R}+\rho\left(v_{1}+v_{2}\right)+\sigma\left(v_{1}^{2}+v_{2}^{2}\right)}, . . \\
& c=(e-a) p=q-p a+p b, . . . . . . .
\end{aligned}
$$

the equation changes into

$$
i=\frac{1}{2}\left(p u^{\prime}-a-b\right)+\frac{1}{2} \sqrt{\left(p u^{\prime}+a-b\right)^{2}+4 c u} .
$$




\section{§. Comparison of the Values of the Work.}

After having obtained a definite expression for the currentstrength $i$, the expressions for $\mathrm{T}_{1}$ and $\mathrm{T}_{2}$ given in (III.) and (V.) are completely defined, so that they may serve for calculating these two magnitudes.

In order to make the formulæ more adapted for comparison, we will introduce a simple sign for the member affected by the small factors $\sigma$ and $\lambda$ by putting

$$
\gamma=\left(\frac{l}{a+i}\right)^{2}\left(\sigma+\frac{\lambda}{b+i}\right) . \quad . \quad . \quad .
$$

The two equations then become

$$
\begin{aligned}
& \mathrm{T}_{1}=\left(\frac{e+i}{(a+i)(b+i} p v_{1}+\gamma v_{1}^{2}\right) i^{2}, \ldots . \\
& \mathrm{T}_{2}=\left(\frac{e+i}{(a+i)(b+i)} p v_{2}-\gamma v_{2}^{2}\right) i^{2} . . .
\end{aligned}
$$

Moreover from (5),

$$
\frac{e+i}{(a+i)(b+i)} p=\frac{\mathrm{R}+\rho\left(v_{1}+v_{2}\right)+\left(\sigma+\frac{\lambda}{b+i}\right)\left(v_{1}^{2}+v_{2}^{2}\right)}{v_{1}-v_{2}} .
$$

We will here again introduce an abbreviated symbol by putting

$$
\delta=\rho\left(v_{1}+v_{2}\right)+\left(\sigma+\frac{\lambda}{b+i}\right)\left(v_{1}^{2}+v_{2}^{2}\right), \quad . \quad .
$$

by which the expression changes into

$$
\frac{e+i}{(a+i)(b+i)} p=\frac{\mathrm{R}+\delta}{v_{1}-v_{2}} \text {. . . . . . }
$$

By using this equation we can put the above two equations for $T_{1}$ and $T_{2}$ in the form

$$
\begin{aligned}
& \mathrm{T}_{1}=\left[(\mathrm{R}+\delta) \frac{v_{1}}{v_{1}-v_{2}}+\gamma v_{1}^{2}\right] i^{2}, \ldots . \\
& \mathrm{T}_{2}=\left[(\mathrm{R}+\delta) \frac{v_{2}}{v_{1}-v_{2}}-\gamma v_{2}^{2}\right] i^{2}, . .
\end{aligned}
$$

Subtracting these equations from one another, we have

$$
\mathrm{T}_{1}-\mathrm{T}_{2}=\left[\mathrm{R}+\delta+\gamma\left(v_{1}^{2}+v_{2}^{2}\right)\right] i^{2} . \quad . \quad .
$$


If we divide the two equations into one another, we get in the first place

$$
\frac{\mathrm{T}_{2}}{\mathrm{~T}_{1}}=\frac{v_{2}}{v_{1}} \cdot \frac{1-\gamma \frac{v_{2}\left(v_{1}-v_{2}\right)}{\mathrm{R}+\delta}}{1+\gamma \frac{v_{1}\left(v_{1}-v_{2}\right)}{\mathrm{R}+\delta}} ;
$$

and if in this we develop the fraction containing the magnitade $\gamma$, in powers of $\gamma$, disregarding members of higher degree than the first, because $\gamma$ contains the factors $\sigma$ and $\lambda$, of which the higher powers have already been disregarded in previous calculations, we arrive at

$$
\frac{\mathrm{T}_{2}}{\mathrm{~T}_{1}}=\frac{v_{2}}{v_{1}}\left(1-\gamma \frac{v_{1}^{2}-v_{2}^{2}}{\mathrm{R}+\delta}\right) . \quad \cdot . \cdot \cdot \cdot
$$

These equations show in a comprehensive manner how the magnitudes $T_{1}$ and $T_{2}$ depend on the velocities of rotation, and on the current-strength also affected by these velocities, and in what relations they stand to each other. The equations can therefore be used to draw conclusions how the working of the machine is to be arranged, so as to obtain the most favourable action under given conditions.

In this latter respect a conclusion is sometimes adduced, which in the year $1840 \mathrm{M}$. H. Jacobi drew as to the action of an older form of electromagnetic machine worked by a voltaic current, and which he thus formulated*:-"The consumption of zine which takes place when the machine is at rest and does no work is double that which takes place when it does the maximum work." This conclusion has been applied in a modified form to the transmission of power by dynamo machines, in which the velocities of rotation of the two machines could be used as the variables for determining the current-strength and the work, and from which the principle has been deduced that the greatest useful effect is obtained when the ratio of the two velocities is as $2: 1$.

It must be observed in this connexion that Jacobi drew his conclusion from somewhat inaccurate assumptions, the first of which is-" The strength of the magnetism excited in soft iron by voltaic currents is proportional to the strength of these currents;" and that therefore no great accuracy can be assigned to his conclusions. It must, on the other hand, be observed that this conclusion refers to a strictly defined process, where a voltaic battery, the work of which is governed by fixed laws, served as the source of power; while, in the transmission of power, other sources may be used the

* Jacobi, Report of the British Association, 1840, and Pogg. Ann. vol. li. p. 358,1840 . 
work of which is at our disposal in various ways. Hence, if we are to make investigations as to the maximum of the work transferred, we must first of all accurately define the conditions to which this maximum refers.

\section{\$4. Determination of the Work $\mathrm{T}_{2}$ when the Velocity $v_{1}$ is supposed to be given.}

In reference to the circumstance last mentioned, that the source of power, which we have to use for driving the first machine, is in various ways at our disposal, we will first make the assumption that the source of power is of such a kind as to do work of any magnitude, so that the work $T_{1}$ which can be expended in the first machine is unlimited. This would be the case, for instance, if a great water-power were at our disposal, which would far exceed the demand even with the greatest possible consumption of work. The question is, How can the greatest work $T_{2}$ be obtained from the second machine under these circumstances?

If $\mathrm{T}_{2}$ is to be large, then from (15) $v_{2}$ and $i$ must be large, and a great value of $i$ presupposes again (12) a great value of the difference $v_{1}-v_{2}$. The two conditions that $v_{2}$ and $v_{1}-v_{2}$ must be great can only be fulfilled when $v_{1}$ is great; and the first condition for a great value of $\mathrm{T}_{2}$ is therefore that $v_{1}$, the velocity of rotation of the first machine, must be as great as possible. In this respect we are confined within certain limits, arising from the mechanical difficulties which oppose the production of very great velocities; and we will accordingly consider the greatest attainable velocity as a given magnitude, and assume that $v_{1}$ is this velocity.

Of the two velocities of rotation, $v_{2}$ only remains undefined; and we may accordingly regard the work $\mathrm{T}_{2}$ as a function of this magnitude. To determine this function we will use the equation already deduced, that is,

$$
\mathrm{T}_{2}=\left[(\mathrm{R}+\delta) \frac{v_{2}}{v_{1}-v_{2}}-\gamma v_{2}^{2}\right] i^{2},
$$

in which we may replace $i$ by its value given in (12). The magnitudes $u$ and $u^{\prime}$, which occur in this latter, are functions of $v_{1}$ and $v_{2}$ in accordance with (6) and (10); and hence also the entire expression for $\mathrm{T}_{2}$ obtained in this way is a function of $v_{1}$ and $v_{2}$. As, further, we must consider $v_{1}$ as given and only $v_{2}$ variable, we may also say that in this way $\mathrm{T}_{2}$ is represented as a function of the single variable $v_{2}$.

In order now to find that value of $v_{2}$ for which $\mathrm{T}_{2}$ is a maximum, we must apply the well-known method of differentiating $\mathrm{T}_{2}$ in respect of $v_{2}$, putting the differential coefficient

Phil. Mag. S. 5. No. 109. Suppl. Vol. 17. $2 \mathrm{~N}$ 
equal to null, and solving the resultant equations in respect of $v_{2}$.

But from the frequent and multifarious occurrence of $v_{2}$ this calculation is tedious, even when the members containing the small values $\sigma$ and $\lambda$ are simplified by introducing approximate values, as is undoubtedly allowable. We shall therefore content ourselves with making the calculation for a special case and with certain omissions, in order to get at any rate an approximate idea of the magnitude of the value $v_{2}$ in question. The constant $c$, which is equal to $(e-a) p$, may, according to the values of $e$ and $a$, be either positive or negative, and thus may also be null in a particular case ; we will assume that this constant has the last-named special value null. The expression for $i$ acquires then the following simple form:-

$$
i=p u^{\prime}-b \text {. }
$$

We will further disregard, in the calculation, all members containing the factors $\rho, \sigma$, and $\lambda$; we have then to put $u^{\prime}$ equal to $\left(v_{1}-v_{2}\right) / R$, and make $y$ and $\delta$ equal to null. The equation which holds for $\mathrm{T}_{2}$ passes then into

$$
\mathrm{T}_{2}=\mathrm{R} \frac{v_{2}}{v_{1}-v_{2}}\left(p \frac{v_{1}-v_{2}}{\overline{\mathrm{R}}}-b\right)^{2} \cdot \text {. . . }
$$

By differentiating this equation in respect of $v_{2}$ we get

$$
\frac{\partial \mathrm{T}_{2}}{\partial v_{2}}=\frac{1}{\left(v_{1}-v_{2}\right)^{2}}\left(p \frac{v_{1}-v_{2}}{\mathrm{R}}-b\right)\left(2 p v_{2}^{2}-3 p v_{1} v_{2}+p v_{1}^{2}-\mathrm{R} b v_{1}\right) \text {. }
$$

If now this differential equation is equal to null, one of the three factors of which the expression on the right hand consists is thus null. The first factor cannot of course be null. The second factor

$$
p \frac{v_{1}-v_{2}}{\mathrm{R}}-b
$$

represents the current-strength $i$, and for our purpose cannot be made equal to null. We must hence make the third factor null, and thus obtain for the deternination of $v_{2}$ the equation

From this follows

$$
2 p v_{2}^{2}-3 p v_{1} v_{2}+p v_{1}^{2}-\mathrm{R} b v_{1}=0 . \text {. . . }
$$

$$
v_{2}=\frac{3}{4} v_{1} \pm \sqrt{\frac{1}{16} v_{1}^{2}+\frac{\widehat{\mathrm{R} b} v_{1}}{2 p}} .
$$

The upper sign before the root gives a value for $v_{2}$ which is greater than $v_{1}$, and which therefore is inadmissible in our case; hence we must use the lower sign, and then by a slight transformation we get the equation

$$
v_{2}=v_{1}\left(\frac{3}{4}-\frac{1}{4} \sqrt{1+8 \frac{\overline{\mathrm{R} b}}{p v_{1}}}\right) . \text {. . . }
$$


This equation shows that the value of $v_{g}$ which corresponds to the maximum of $\mathrm{T}_{2}$ is not equal to $v_{1} / 2$, as the above law states, but approaches this value with increase of $v_{1}$.

\section{$\S 5$. Determination of the work $\mathrm{T}_{2}$ when the work $\mathrm{T}_{1}$ is given.}

The second assumption which we can make in regard to the source of power which drives the first machine, may be that it can only perform a work $\mathrm{T}_{1}$ of definite magnitude. The question then arises, Does the work $\mathrm{T}_{2}$ to be gained from the second machine depend on the working of the machines?

It is here to be observed that, if $T_{1}$ is given, only one of the velocities of rotation $v_{1}$ and $v_{2}$ is to be considered as independent. As three equations, (III.), (V.), and (3), are given between the five magnitudes $v_{1}, v_{2}, \mathrm{~T}_{1}, \mathrm{~T}_{2}$, and $i$, from two of these magnitudes the other three can be determined. We will consider $v_{1}$ as that magnitude which, along with $\mathrm{T}_{1}$, serves to define the other three values.

In order to define $i$ as a function of ' $\mathrm{T}_{1}$ and $v_{1}$ we use the equation (III.), that is,

$$
\mathrm{T}_{1}=\left[\frac{e+i}{(a+i)(b+i)} p v_{1}+\left(\frac{l}{a+i}\right)^{2}\left(\sigma+\frac{\lambda}{b+i}\right) v_{1}^{2}\right] i^{2},
$$

which we may bring into the following form:-

$$
\frac{(e+i) i^{2}}{(a+i)(b+i)}=\frac{\mathrm{T}_{1}}{p v_{1}}-\left(\frac{l i}{a+i}\right)^{2}\left(\sigma+\frac{\lambda}{b+i}\right) \frac{v_{1}}{p} .
$$

The solution of this equation in respect of $i$ is rendered more difficult by the second member on the right hand, which contains $i$ in the denominator in a higher power than the member on the left hand. It is to be observed, however, that the member on the right hand is affected by the factors $\sigma$ and $\lambda$, which are so small that we may disregard magnitudes in reference to them which are of higher than the first order. This fact may be utilized in treating the equation. We first of all determine that value of $i$ which results from the equation when we disregard the member containing the factors $\sigma$ and $\lambda$. If we denote this value for distinction by $i^{\prime}$, the equation for its determination is

$$
\frac{\left(e+i^{\prime}\right) i^{2}}{\left(a+i^{\prime}\right)\left(b+i^{\prime}\right)}=\frac{\mathrm{T}_{1}}{p v_{1}} \text {. . . . . . }
$$

The value of $i^{\prime}$ resulting from this can only differ from $i$ by a magnitude of the order of the factors $\sigma$ and $\lambda$; and if we return to $(26)$, and in the member affected by these factors replace $i$ by the value $i^{\prime}$, the expression on the right hand only changes by a magnitude which in reference to $\sigma$ and $\lambda$ is of the second order, and therefore may be neglected. Henco $2 \mathrm{~N} 2$ 
instead of (26) we construct the equation

$$
\frac{(e+i) i^{2}}{(a+i)(b+i)}=\frac{\mathrm{T}_{1}}{p v_{1}}-\left(\frac{l i^{\prime}}{a+i^{\prime}}\right)^{2}\left(\sigma+\frac{\lambda}{b+i^{\prime}}\right) \frac{v_{1}}{p} .
$$

In this we will introduce a simplifying expresion $z$ with the meaning

$$
z=\frac{\mathrm{T}_{1}}{p v_{1}}-\left(\frac{l i^{\prime}}{a+i^{\prime}}\right)^{2}\left(\sigma+\frac{\lambda}{b+i^{\prime}}\right) \frac{v_{1}}{p}, \ldots .
$$

by which the equation changes into

$$
\frac{(e+i) i^{2}}{(a+i)(b+i)}=z \text {. . . . . . }
$$

The two equations (27) and (30) are of the same form, so that the solution of one at once gives that of the other. Selecting equation (30) for treatment, we bring it by multiplication with $(a+i)(b+i)$ into the form of an ordinary cubical equation, that is,

$$
i^{3}-(z-e) i^{2}-(a+b) z i-a b z=0, \quad . \quad .
$$

and apply to this the well-known method of solving cubical equations. Only one of the three roots thereby obtained can be used on account of the sign, and this is determined as follows:-Let $\phi$ be the angle between 0 and $\pi$ which satisfies the equation

$$
\cos \phi=\frac{(z-e)^{3}+\frac{9}{2}(a+b)(z-e) z+\frac{27}{2} a b z}{\sqrt{\left[(z-e)^{2}+3(a+b) z\right]^{3}}} ; .
$$

$i$ will be represented by the following expression:-

$$
i=\frac{1}{3}(z-e)+\frac{2}{3} \sqrt{(z-e)^{2}+3(a+b) z} \cdot \cos \frac{\phi}{3} . \quad .
$$

In reference to the $\operatorname{cosin} \theta$ which occurs as a factor of the last member, it follows from equation (32) that while $z$ increases from 0 to $\infty$, the angle $\phi$ decreases from $\pi$ to 0 , from which it follows that $\phi / 3$ decreases from $\pi / 3$ to 0 , and accordingly $\cos (\phi / 3)$ increases from $\frac{1}{2}$ to 1 . Here it is to be observed that the first increase is pretty rapid, so that in the values of $z$ which occur in practice, $\cos (\phi / 3)$ cannot be very different from 1 .

Equations (32) and (33) may also be used in a twofold manner. If $z$ is replaced in them by $\mathrm{T}_{1} / p v_{1}$, they form a solution of equation (27), and give that approximate value of $i$ which has been designated above by $i^{\prime}$. By replacing it in (29) we obtain the value $z$, and, knowing this, we can by another application of equations (32) and (33) calculate the stricter value of $i$.

After determining in this way the strength $i$, the first of the above-named three magnitudes, there is no difficulty with the two others.

To determine $v_{2}$ equation (5) can be used. This is an 
equation of the second order, and could be solved as such; yet here also the fact that the square of $v_{2}$ only occurs with the small factors $\sigma$ and $\lambda$ may be used for simplifying the calculation. For this the equation may be written in the following form:-

$$
v_{2}=\frac{\frac{e+i}{(a+i)(b+i)} p v_{1}-\mathrm{R}-\rho v_{1}-\left(\sigma+\frac{\lambda}{b+i}\right)\left(v_{1}^{2}+v_{2}^{2}\right)}{\frac{e+i}{(a+i)(b+i)} p+\rho} .
$$

In this we may omit the last term of the numerator, and from the equation thus abbreviated calculate an approximate value of $v_{2}$. This value can then be introduced into that last member, and thus the more accurate value of $v_{2}$ determined by a second calculation.

When this magnitude $v_{2}$ is known, the determination of the last magnitude, with which we are specially concerned (that is, the work done by the second machine $\mathrm{T}_{2}$ ), follows directly, as equation (20) can be used, which, after replacing $\gamma$ and $\delta$ by their values, may be written as follows:-

$$
\mathrm{T}_{2}=\mathrm{T}_{1}-\left[\mathrm{R}+\rho\left(v_{1}+v_{2}\right)+\left(1+\frac{l^{2}}{(a+i)^{2}}\right)\left(\sigma+\frac{\lambda}{b+i}\right)\left(v_{1}^{2}+v_{2}^{2}\right)\right] i^{2} \text {. }
$$

We see from this equation, in which the negative member on the right hand has the factor $i^{2}$, that $\mathrm{T}_{2}$ is greater (that is, is nearer the given value $T_{1}$ ) the smaller $i$ is. But as, from (33), $i$ decreases simultaneously with $z$, and as, further, the chief member of the expression for $z$ in (29) is the fraction $\mathrm{T}_{1} / p v_{1}$, and therefore decreases when $v_{1}$ increases, $i$ also decreases when $v_{1}$ increases; and hence we get the result for $\mathrm{T}_{2}$, that its value is greater as $v_{1}$ is greater; and it is in fact known from practice that, in order to make $T_{2}$ as great as possible in reference to $T_{1}$, the velocity of rotation of the first machine must be increased to the utmost possible limit.

According to (32), the velocity $v_{2}$ of the second machine is greater the greater $v_{1}$ and the smaller $i$ becomes. But as, from the preceding, with a given value of $T_{1}$, a decrease of $i$ is connected with an increase of $v_{1}$, it follows from (34) that $v_{2}$ increases with an increase of $v_{1}$ in a still greater ratio than $v_{1}$ itself.

It will be clear from what has been said that in this case, in which $\mathrm{T}_{1}$ is considered as given, there can be no question of a definite ratio between $v_{2}$ and $v_{1}$ for which $\mathrm{T}_{2}$ would be a maximum.

In this paragraph, from the magnitudes $\mathrm{T}_{1}$ and $v_{1}$ the three other magnitudes $i, v_{2}$, and $\mathrm{T}_{2}$ were determined; and in the previous paragraph the magnitudes $i$ and $\mathrm{T}_{2}$ from the magnitudes $v_{1}$ and $v_{2}$. In like manner a series of other calculations 
may be made with our equations, by selecting any two of the five magnitudes and determining the other three from them. But we will content ourselves with the above instances of oalculations, which refer to two of the most important cases which occur in practice.

The results obtained by calculation are perhaps rather more complicated than is desirable for practical use ; it may, however, be observed that the aim was to deduce the results from the principal equations in such a manner that only higher powers of the small magnitudes $\sigma$ and $\lambda$ are neglected. If we are content with a less degree of accuracy, the calculations and the form of their results may be simplified. Moreover, when the results derived from the principal equations are once known, it will not be difficult to form approximate formulæ, which give the results with sufficient accuracy for certain practical purposes, and are more convenient for use. But I will not here enter on such external transformations, as they are outside the object of the present paper.

Bonn, January 1884.

EnRata in the Paper on the Theory of Dynamo-electrical Machines

Page Line

4722 for magnets read machines

" 38 after coil add but may be at rest

4921 after conductor add $\sigma$

506 for positions read portions

517 for two branch circuits at rest read two collateral branch circuits

5410 for branch read neighbouring

9 16 for the potential read these potentials

554 for $\mathrm{E}_{2}$ read $\mathrm{E}_{1}$

$5825,26,28,36$, and 37 , for numerator read denominator

" 16 for ones for the read quantities of

" 17 after work add T

" 18 for time T read unit of time

" 8 for opposite read the same

12328 for the change.... we have read the component of the original magnetic moment which falls in the altered direction. The original magnetic moment is

1262 for numerator read denominator

12820 for $\frac{q}{h+i}$ read $\frac{q}{b+i}$

"30 28 delete external

$130 \quad 39$ after in add (33) and

1318 for no read small

" 40 for $\frac{A i^{\prime}}{1+a i}$ read $\frac{A i}{1+\infty i}$

13222 for magnet read machine

" 29 for acts read acting

" 30 delete and

1.33 5 for determine read introduce

" 25 for $i$ read $i_{1}$ 